\section{THEORY OF THE STRUCTURE OF CLAY MINERALS}

\section{TAILED X-RAY REFLEXIONS FROM RANDOM LAYER LATTICES}

$S^{\circ}$

ME substances, such as chrysotile and graphite, appear to possess a layer structure in which the comparatively perfect layers are displaced by amounts that are either random (graphite) or effectively random for some reflexions (chrysotile). In the reciprocal-space representation, these reflexions are represented by rods perpendicular to the layers, the cross-section of the rods depending on the size of the layers, and the variation of intensity along them depending on the variation of the structure amplitude of a single layer. Warren ${ }^{1}$ has calculated the line profile of such reflexions on powder photographs, replacing the true variation of intensity across the rod by a Gaussian distribution. Patterson ${ }^{2}$ has shown in the related problem of line-broadening by small particles that this is not a very good approximation, and it therefore seemed desirable to derive expressions for the line profile without the use of it.

The intensity of reflexion from a crystal is

$$
I(\mathbf{H})=\Sigma_{j} \Sigma_{j^{\prime}} F_{j} F^{*}{ }_{j^{\prime}} \exp \left\{2 \pi i\left(\mathbf{r}_{j^{\prime}}-\mathbf{r}_{j}\right) . \mathbf{H}\right\},
$$

where $\mathbf{H}$ is the position vector in reciprocal space and $F_{j}$ and $\mathbf{r}_{j}$ are the structure amplitude and position vector of the $j$ th unit cell ${ }^{3}$. For reflexions for which the phase relation between successive layers is effectively random, the intensity will be simply $N$ times that of a single layer, where $N$ is the number of layers, and equation 1 becomes

$I(\mathbf{H})=N \Sigma_{j_{1}} \Sigma_{j_{\mathbf{s}}} \Sigma_{j_{1}} \Sigma_{j_{\mathbf{s}}} F_{j} F^{*_{j^{\prime}}} \exp \left\{2 \pi i\left(\mathbf{r}_{j^{\prime}}-\mathbf{r}_{j}\right) . \mathbf{H}\right\},(2)$

where $\mathbf{r}_{j}$ is now the position vector within a layer. The intensity will be large when

$$
\mathbf{H}=h \mathbf{a}^{\prime}+k \mathbf{b}^{\prime}+w \mathbf{c}^{\prime},
$$

where $\mathbf{c}^{\prime}$ is a unit vector perpendicular to the layers, $\mathbf{a}^{\prime}$ is of length $1 / a \sin \gamma$ and perpendicular to $\mathbf{b}$ and $\mathbf{c}^{\prime}, \mathbf{b}^{\prime}$ is of length $1 / b \sin \gamma$ and perpendicular to a and $\mathbf{c}^{\prime}, h$ and $k$ are integers, and $w$ is arbitrary. (In general, $\mathbf{a}^{\prime}$ and $\mathbf{b}^{\prime}$ do not coineide with $\mathbf{a}^{*}$ and $\mathbf{b}^{*}$.) Let $\mathbf{H}=\mathbf{S}_{0}+\boldsymbol{\rho}$, where $\mathbf{S}_{0}=h \mathbf{a}^{\prime}+h \mathbf{b}^{\prime}$, and let $(x, y),(u, v)$ be the components of $\mathbf{r}$ and $\mathbf{p}$ in the direction of $S_{0}$ and in the direction perpendicular to $S_{0}$ and $c^{\prime}$. Then in equation (2) $\mathbf{r}_{j} . S_{0}$ is an integer, and may be dropped from the exponentials, and $\mathbf{r}_{j} \cdot \mathbf{p}$ changes only slightly in going from one unit cell to the next, so that the summations may be replaced by integrations over the area of the layer. Then

$$
I(u, v, w)=N F^{2}(w) c C^{-1} \int_{A^{\prime}} \int_{A} \exp \left\{2 \pi i \left[\left(x^{\prime}-x\right) u+\right.\right.
$$$$
\left.\left.\left(y^{\prime}-y\right) v\right]\right\} d A d A^{\prime}
$$

where $C$ is the area of the face of the unit cell containing $\mathbf{a}$ and $\mathbf{b}$, and $c$ is the interlayer spacing. Except for certain geometrical factors, the intensity of reflexion as a function of $S=2 \sin \theta / \lambda$ is got by integrating $I(u, v, w)$ over the space between two spheres of radii $S$ and $S+d S$, but to a sufficient approximation these may be replaced by tangent paraboloids. The equation of the paraboloid making the closest contact with the sphere $S^{2}=\mathbf{H} . \mathbf{H}=$ $S_{0}^{2}+2 u S_{0}+u^{2}+v^{2}+w^{2}$ in the important region $u, v \sim 0$ is

$$
S^{2}=S_{0}^{2 *}+2 u S_{0}+w^{2} \text {. }
$$

Let $\sigma=\left(S^{2}-S_{0}{ }^{2}\right) / 2 S_{0}$. The intensity of reflexion as a function of $\sigma$ is given by the integral of $I(u, v, w)$ $d u d v d w$ over the space between the two spheres or, since the volume element $d u d v d w$ corresponds to $d v d w d \sigma, \mathrm{by}$

$$
\begin{gathered}
I(\sigma) d \sigma=N c C^{-1} \int_{-\infty}^{+\infty} \int_{-\infty}^{+\infty} \int_{A^{\prime}} \int_{A} F^{2}(w) \exp \left\{2 \pi i \left[\left(x^{\prime}-x\right) \sigma-\right.\right. \\
\left.\left.\left(x^{\prime}-x\right) w^{2} / 2 S_{0}+\left(y^{\prime}-y\right) v\right]\right\} d A d A^{\prime} d v d w d \sigma .
\end{gathered}
$$

The integrals with respect to $v$ and $y^{\prime}$ are singular, the double integral giving

$$
\begin{aligned}
I(\sigma)=N c C^{-1} \int_{-\infty}^{+\infty} \int_{x^{\prime}} \int_{A} F^{2}(w) \exp \left\{2 \pi i \left[\left(x^{\prime}-x\right) \sigma-\right.\right. \\
\left.\left.\left(x^{\prime}-x\right) w^{2} / 2 S_{0}\right]\right\} d A d x^{\prime} d w,
\end{aligned}
$$

or, with $x^{\prime}-x=t$ and $A(t)=\iint d x d y$ over the area common to the layer and its 'ghost' shifted a distance $t$ parallel to $\mathbf{S}_{0}$ (compare ref. $4, \mathrm{p}$. 37),

$$
\begin{aligned}
& I(\sigma)=N c C^{-1} \int_{-\infty}^{+\infty} \int_{-\infty}^{+\infty} F^{2}(w) A(t) \\
& \exp \left\{2 \pi i\left[\sigma t-w^{2} t / 2 S_{0}\right]\right\} d w d t .
\end{aligned}
$$

If the variation of $F^{2}$ with $w$ be neglected, the integration with respect to $w$ is of the Fresnel type, giving

$$
\begin{aligned}
I(\sigma)=N F^{2} c C^{-1}\left(S_{0} / 2\right)^{1 / 2} \int_{-\infty}^{+\infty} A(t)|t|^{-1 / 2} \\
(\cos 2 \pi \sigma t+\sin 2 \pi \sigma|t|) d t .
\end{aligned}
$$

This is analogous to the expression for the line profile for a normal $h k l$ reflexion from a small crystal,

$$
I(s)=F^{2} U^{-1} \int_{-\infty}^{+\infty} V(t) \cos 2 \pi s t d t,
$$

where $s=S-S_{0}$ and $V(t)$ is the volume common to the crystal and its 'ghost' shifted a distance $t$ perpendicular to the $h k l$ planes (ref. 4, p. 41; ref. 5). The apparent particle size for the normal reflexion (equation 9) is easily obtained in the form

$$
\varepsilon=V^{-1} \int V(t) d t
$$

but no corresponding expression can be obtained for the tailed reflexion (equation 8), since (i) $\int I(\sigma) d \sigma$ does not converge, and (ii) the position and height of the maximum of $I(\sigma)$ cannot be expressed in simple form. The nearest analogue is perhaps the ratio of the slope $\partial I / \partial \sigma$ to the actual value of $I(\sigma)$ for $\sigma=0$ :

$$
\varepsilon^{\prime}=\left.2 \pi \int_{-\infty}^{+\infty} A(t)|t|^{1 / 2} d t\left|\int_{-\infty}^{+\infty} A(t)\right| t\right|^{-1 / 2} d t .
$$

This may be evaluated for simple layer shapes; for a square, with sides of length $X$ making angles with $\mathbf{S}_{0}$ the positive values of the cosines of which are $m \geqslant n$, it is

$$
\varepsilon^{\prime}=2 \pi X(7 m-3 n) / 7 m(5 m-n) \fallingdotseq 1 \cdot 26 X,
$$

and for a circle of diameter $D$ it is

$$
\varepsilon^{\prime}=\frac{3 \pi D}{10}\left[\frac{\Gamma(5 / 4)}{\Gamma(7 / 4)}\right]^{2} \fallingdotseq 0.924 D \fallingdotseq 1 \cdot 04 \sqrt{ }\left(\pi D^{2} / 4\right) \text {. }
$$




$$
\begin{aligned}
& I(\sigma) d \sigma=N F^{2} c C^{-1} A\left(2 S_{0}\right)^{1 / 2} \sigma^{-1 / 2} d \sigma, \\
& I(S) d S=N F^{2} c C^{-1} A .2 S\left(S^{2}-S_{0}{ }^{2}\right)^{-1 / 2} d S,
\end{aligned}
$$

where $A$ is the area of a layer; and for $\sigma$ large and negative it is zero. For small $\sigma$, equation (8) may be expanded as a power series in $\sigma|t|$ and integrated term by term for values of $A(t)$ corresponding to various layer shapes. Preliminary results indicate differences of a few per cent from Warren's curve, especially for $\sigma$ negative.

Without a knowledge of the crystal structure it appears difficult to take the variation of $F^{2}$ with $w$ into account in evaluating the line profile. From an experimental knowledge of the line profile it might be possible to work back to the crystal structure ; a Fourier transformation applied to equation (7) leads to

$$
\begin{gathered}
\int_{-\infty}^{+\infty} F^{2}(w) \exp \left\{-\pi i w^{2} t / S_{0}\right\} d w= \\
\frac{C}{N c A(t)} \int_{-\infty}^{+\infty} I(\sigma) \exp \{-2 \pi i \sigma t\} d \sigma,
\end{gathered}
$$

the left-hand side of which is easily expressed in terms of $h, k, t$ and the atomic co-ordinates. The $x$ and $y$ components of the interatomic vectors appear linearly, but the $z$ components occur squared.

This work forms part of an investigation of the diffraction of X-rays by imperfect structures, for which an apparatus grant has been received from the Royal Society.

\section{Viriamu Jones Laboratory,}

$$
\text { A. J. C. WILson }
$$

University College,

Cardiff.

Dec. 2.

${ }^{1}$ Warren, B. E., Phys. Rev., 59, 693 (1941).

2 Patterson, A. L., Phys. Rev., 56, 978 (1939).

${ }^{3}$ Wilson, A. J. C., Proc. Roy. Soc., A, 180, 277 (1942).

4 Wilson, A. J. C., "X-Ray Optics" (Methuen, London, 1948)

- Stokes, A. R., and Wilson, A. J. C., Proc. Camb. Phil. Soc., 38, 313 (1942).

\section{BANDED X-RAY REFLEXIONS FROM CLAY MINERALS}

THE banded character of many of the X-ray 'lines' from such clay minerals as montmorillonite and halloysite has been attributed to their structures being equivalent to a random stacking of two-dimensional silicate sheets ${ }^{1}$. The relevant theory has been developed by Warren ${ }^{2}$, who introduced simplifying assumptions in order to obtain explicit expressions for the intensity distribution. We have sought a more general expression than Warren gives, and have avoided certain of his approximations by expressing the results in a form requiring numerical integration.

A finite two-dimensional crystal with axes $a_{1} a_{2}$ is represented in reciprocal space, $R$-space, by a lattice with axes $b_{1} b_{2}$, the points of which are diffused parallel to the $b_{1} b_{2}$ plane in a manner depending on the erystal size and shape, and normal to this plane in accordance with the cell structure-factor $F$. The diffracted intensity is determined by the inter. section in $R$-space of the sphere of reflexion with these diffused lines. Allowance must also be made for the fact that powders and not single crystals are used.

For an infinite two-dimensional crystal sheet of periodic electron density $P_{\infty}(a)$, (Ewald's notation ${ }^{3}$ ), the Fourier transform consists of straight lines $h k$ of linear density $F^{\prime}(b) / A_{a}$, where $F(b)$ is the structure

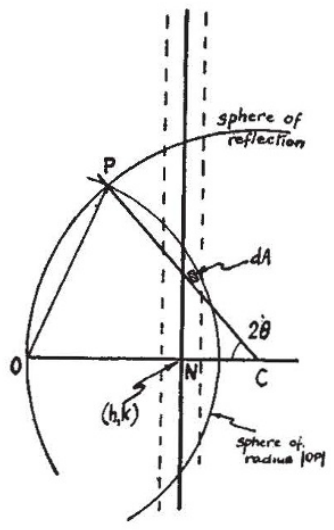

factor and $A_{a}$ is the cell area. For a finite crystal of shape $s(a)$,

$$
\rho_{8}(a)=\rho_{\infty}(a) s(a),
$$

and the Fourier transform of $p_{s}(a)$ is $F(b) S(b) / A_{a}$. The shape transform, $S(b)$, is the diffraction pattern of $s(a)$, which for two-dimensional crystal fiakes is the diffraction pattern observed optically. $F S / A_{a}$ represents the scattered X-ray amplitude, and the corresponding intensity expression is $F F^{*} S S^{*} / A_{a^{2}}$, where * signifies the conjugate complex.

The diagram represents the diffused line associated with a lattice point $N$ in $R$-space and shows the condition for reflexion in direction $C P$, where $P$ is a point on the sphere of reflexion of radius $1 / \lambda$. For randomly arranged crystals, the average reflected intensity is obtained by averaging over a sphere of radius $|O P|=2 \sin \theta / \lambda$, and is equal to

$$
\left[\int F F^{*} S S^{*} d A\right] / A_{a^{2}} 4 \pi(2 \sin \theta)^{2} / \lambda^{2},
$$

where $d A$ represents a surface element of the sphere of radius $|O P|$. This has to be multiplied by the usual electronic and polarization factors,

$$
\frac{e^{4} I_{0}}{m^{2} c^{4}}\left(\frac{1+\cos ^{2} 2 \theta}{2}\right) \text {. }
$$

If $d E(2 \theta)$ is the energy reflected between directions $2 \theta$ and $2(\theta+d \theta)$, that is, in $d w=4 \pi \sin 2 \theta \cdot d \theta$, then the reflected intensity $I(2 \theta)=d E / 4 \pi \sin 2 \theta \cdot d \theta$ and $d E / d(2 \theta)=2 \pi I(2 \theta) \cdot \sin 2 \theta$. Only a fraction $1 / 2 \pi R$ $\sin 2 \theta$ of this energy is recorded per unit length of line in a camera of radius $R$. Thus the reflected energy in angular range $d(2 \theta)$ per unit length of line on the film is given by

$\frac{d E}{d(2 \theta)}=\frac{I_{0} e^{4} \lambda^{2}}{m^{2} c^{4} R A_{a^{2}}}\left(\frac{1+\cos ^{2} 2 \theta}{32 \pi \sin ^{2} \theta}\right) \int F F^{*} S S^{*} d A$. (1)

The integral in this expression can be calculated numerically for crystal flakes of simple shape and of known layer structure. A close approximation to integration over the spherical surface is obtained by replacing the sphere by a series of thin cylindrical strips running parallel to the $b_{1} b_{2}$ plane. Provided $F F^{*}$ does not vary greatly across the diffused line in $R$-space, then $F^{*} F^{*}$ may be treated as constant for integration along each cylindrical strip. With simple crystal shapes an algebraic solution of $\int S S * d A$ may be obtained for each strip integration; the results of these calculations will be published shortly. For the complete integration, however, the variation of $F F^{*}$ along the diffused line in $R$-space 